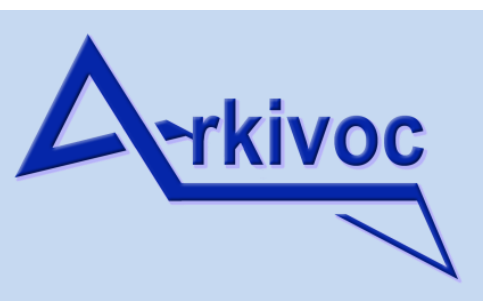

Free to Authors and Readers
A Platinum Open Access Journal for Organic Chemistry

Paper

Arkivoc 2022, part iv, 0-0

DOAJ Seal

\title{
Indole macrocycles formed by diindolylmethane ring closure
}

\author{
Bambang Purwono, Naresh Kumar, and David StC. Black* \\ School of Chemistry, UNSW Sydney, Sydney, NSW 2052, Australia
}

Email: d.black@unsw.edu.au

Received 12-15-2021

Accepted 01-07-2022

Published on line 01-11-2022

\section{Abstract}

Indole containing macrocyclic compounds can be formed from suitable diindolyl diesters and diamides by acidcatalysed reaction with formaldehyde to generate a 2,2'-linkage in the ring closure step. The alternative protocol in which macrocyclisation is attempted by lactone or lactam formation from starting 2,2'diindolylmethane systems was unsuccessful in our examples.<smiles></smiles><smiles></smiles><smiles></smiles>

Keywords: Indoles, macrocycles, diindolylmethanes, lactones, lactams, formaldehyde 


\section{Introduction}

Macrocyclic compounds in general are of increasing interest because of their applications in supramolecular chemistry. ${ }^{1,2}$ Indoles can be incorporated into a wide range of macrocyclic ring systems, ${ }^{3}$ and some of the resulting macrocycles, such as the bis(indolyl)maleimides, show potent biological and antibiotic activity. ${ }^{4-8}$ Most commonly the resulting macrocycles are imines and the ring closure step is carried out by the reaction of indole carbaldehydes with primary amines. ${ }^{9-13}$ Another major approach to indole containing macrocycles involves the construction of indolylmethylene cyclic oligomers, the calixindoles, formed by acid-catalysed reactions of activated indoles with aryl aldehydes, ${ }^{14,15}$ activated indoles with hydroxymethylindoles, ${ }^{16,17}$ or hydroxymethylindoles alone with extrusion of formaldehyde. ${ }^{18}$ In special circumstances, direct oxidative dimerization of 2,7'-biindolyl compounds can generate macrocyclic tetraindolyls, the indorphyrins. ${ }^{19}$

\section{Results and Discussion}

\section{Synthesis of macrocycle precursors}

We have previously shown that indolylesters can be prepared by the reaction of trichloroacetylindoles with alcohols. ${ }^{20}$ Suitable precursors for macrocyclic compounds would be diindolyl diesters or diamides. Therefore two equivalents of the 7-trichloroacetylindole $\mathbf{1}^{21}$ were reacted with one equivalent of ethylene glycol in acetone containing potassium carbonate to give the diindolyl diester $\mathbf{2}$ in $75 \%$ yield (Scheme 1). We have also shown that 2,2'-diindolylmethanes can be readily prepared from 3,7-disubstituted-4,6-dimethoxyindoles by the acid-catalysed reaction with formaldehyde or aryl aldehydes. ${ }^{10,15,20,22}$ In general, alkyl aldehydes either fail to react or follow other paths. Consequently the diindolyl diester $\mathbf{2}$ was heated under reflux with formaldehyde in methanol containing concentrated hydrochloric acid, but no reaction occurred and the starting material remained intact.<smiles>[R]c1c[nH]c2c(C(=O)C(Cl)(Cl)C(F)(F)Cl)c(OC)cc(OC)c12</smiles>

1

$$
\mathrm{R}=4-\mathrm{ClC}_{6} \mathrm{H}_{4}
$$
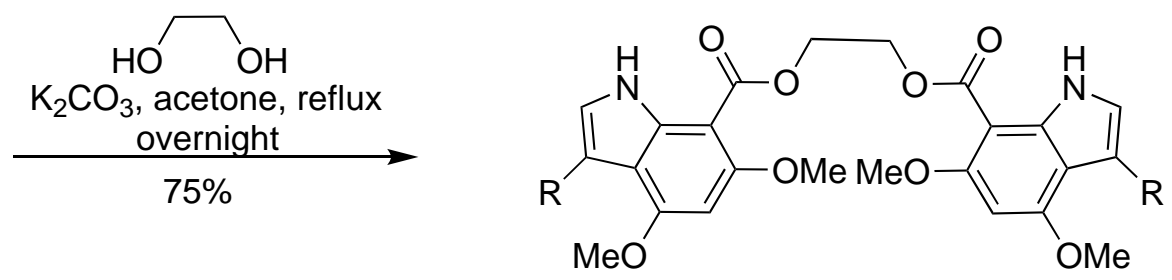

2
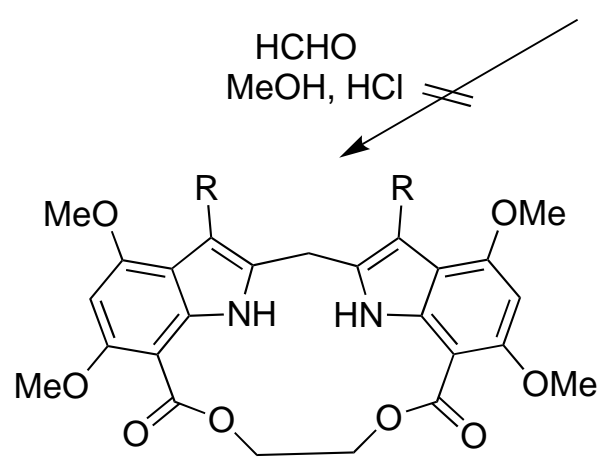

Scheme 1. Formation of diindolyl diester $\mathbf{2}$ by reaction of 7-trichloroacetylindole 1 with ethyleneglycol. 
The failure to form the macrocycle $\mathbf{3}$ was presumably caused by the necessity for the normal hydrogen bonding between the indole $\mathrm{NH}$ and the 7-carbonyl oxygen atom to be broken. This hydrogen bonding would have an effect on the orientation of the ester $\mathbf{2}$, which would discourage macrocyclisation. This result indicates that a longer linkage between the two ester groups is required for macrocyclisation. Therefore, indole $\mathbf{1}$ was reacted with tetraethylene glycol in acetone containing potassium carbonate to give the diindolyl diester $\mathbf{4}$ in $56 \%$ yield (Scheme 2). While in our hands this compound could not be obtained analytically pure, it was nevertheless reacted with formaldehyde to give the 24-membered macrocyclic diindolylmethane 5 in $82 \%$ yield. Furthermore, a similar reaction with 4-chlorobenzaldehyde gave the related macrocycle 6 in $53 \%$ yield.

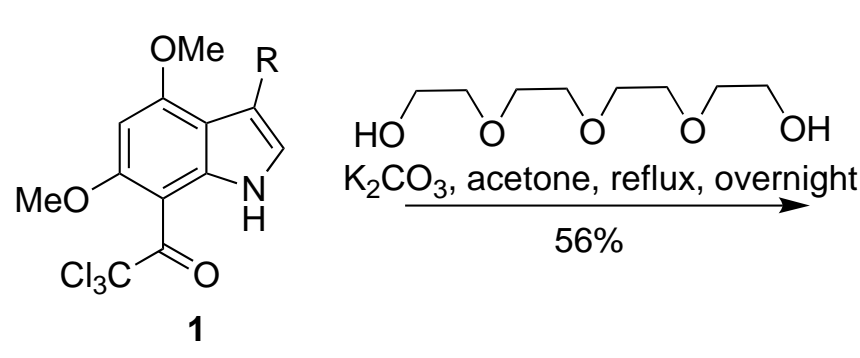

$\mathrm{R}=4-\mathrm{ClC}_{6} \mathrm{H}_{4}$

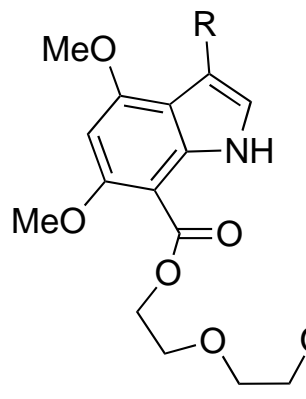

\section{$\mathrm{R}^{1} \mathrm{CHO}$}

$\mathrm{HCl}, \mathrm{MeOH}$,

$3 \mathrm{~h}$ - overnight $\downarrow$

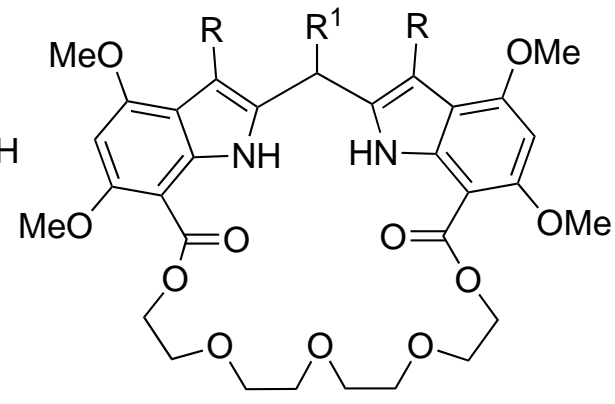

$5 \quad \mathrm{R}^{1}=\mathrm{H} \quad(82 \%)$

$6 \mathrm{R}^{1}=4-\mathrm{ClC}_{6} \mathrm{H}_{4} \quad(53 \%)$

Scheme 2. Formation of diester $\mathbf{4}$ and macrocyclic diindolylmethanes $\mathbf{5}$ and $\mathbf{6 .}$

These results indicate that the length of the ether linker is an important factor to achieve a suitable distance between each indole $\mathrm{C} 2$ position and to take into account the presence of hydrogen bonding between the indole $\mathrm{NH}$ and the 7-carbonyl $\mathrm{O}$ atom. In contrast to the success of macrocyclization through diindolylmethane formation, reaction of the preformed 2,2'-diindolylmethane-7,7'-di(trichloroacetyl) compound $\mathbf{7}^{21}$ with tetraethylene glycol failed to generate any macrocyclic product $\mathbf{5}$ in a complex reaction mixture. Tentatively, the failed ring closure could be attributed to a lack of sufficient flexibility in the diindolylmethane linkage. Therefore, this route cannot be recommended for macrocyclization.

The diindolylmethane macrocyclization protocol was extended to an investigation of diindolyl diamides. Treatment of two equivalents of 7-trichloroacetylindole 1 with one equivalent of diethylenetriamine in acetonitrile at room temperature gave the diindolyl diamide $\mathbf{8}$ in 95\% yield. Reaction of compound 8 with 
formaldehyde in methanol containing concentrated hydrochloric acid then afforded the 18-membered macrocyclic diindolylmethane $\mathbf{1 1}$ in $74 \%$ yield (Scheme 3 ).<smiles>[R]c1c[nH]c2c(C(=O)C(Cl)(Cl)Cl)c(OC)cc(OC)c12</smiles><smiles>OCCOCCOCCOCCO</smiles>
$\mathrm{K}_{2} \mathrm{CO}_{3}$, acetone, reflux, overnight $56 \%$ $\mathrm{R}=4-\mathrm{ClC}_{6} \mathrm{H}_{4}$<smiles>[R]c1c[nH]c2c(C(=O)OCCOCCOCCOCCOC(=O)c3c(OC)cc(OC)c4c([R])c[nH]c34)c(OC)cc(OC)c12</smiles>

$\mathrm{R}^{1} \mathrm{CHO}$ $\mathrm{HCl}, \mathrm{MeOH}$, $3 \mathrm{~h}$ - overnight $\downarrow$<smiles>[R]c1c(C([R])c2[nH]c3c(C(=O)OC)cc(OC)c(OC)c3c2[R])[nH]c2c(C(=O)OCCOCCOCCOCCOC)c(OC)cc(OC)c12</smiles>

$5 \quad \mathrm{R}^{1}=\mathrm{H} \quad(82 \%)$

$6 \mathrm{R}^{1}=4-\mathrm{ClC}_{6} \mathrm{H}_{4} \quad(53 \%)$

Scheme 3. Formation of diamide $\mathbf{8}$ and macrocyclic diindolylmethane $\mathbf{1 1 .}$

A similar reaction of indole 1 with triethylenetriamine gave diamide $\mathbf{9}$ in $60 \%$ yield and subsequent reaction of this product with formaldehyde in methanol containing concentrated hydrochloric acid afforded the 21-membered macrocyclic diindolylmethane $\mathbf{1 2}$ in $80 \%$ yield (Scheme 4). However, in contrast to the synthesis of the macrocyclic diester 6 , attempts to obtain the arylmethine linked macrocycle 14 by reaction of the diindolyl diamide $\mathbf{9}$ with 4-chlorobenzaldehyde failed, and only complex product mixtures were obtained. Several factors could be responsible for the failure to form the macrocycle 14. 4-Chlorobenzaldehyde is less reactive than formaldehyde and also has a greater steric presence. Also formation of a 21-membered ring in the case of compound $\mathbf{1 4}$ would be less accessible than formation of a 24-membered ring in the case of compound 6.

Also, a similar reaction of indole 1 with 2,2'-(ethylenedioxy)bis(ethylamine) generated the diamide 10 in $81 \%$ yield, and further reaction of this compound with formaldehyde in methanol containing concentrated hydrochloric acid afforded the 21-membered macrocyclic diindolylmethane $\mathbf{1 3}$ in $90 \%$ yield (Scheme 5). Once again, reaction of the 2,2'-diindolylmethane-7,7'-di(trichloroacetyl) compound 7 with 2,2'-(ethylenedioxy)bis(ethylamine) failed to generate any macrocyclic product 13 in a complex reaction mixture. 
<smiles>[R]c1c[nH]c2c(C(=O)C(Cl)(Cl)Cl)c(OC)cc(OC)c12</smiles><smiles>CNCCNCCN</smiles><smiles>[R]c1c[nH]c2c(C(=O)NCCNC)c(OC)cc(OC)c12</smiles><smiles>[R]c1c[nH]c2c(C(=O)NCCNCC)c(OC)cc(OC)c12</smiles>

$$
\mathrm{R}=4-\mathrm{ClC}_{6} \mathrm{H}_{4}
$$

\section{$\mathrm{RCHO}$} $\mathrm{HCl}, \mathrm{MeOH}$

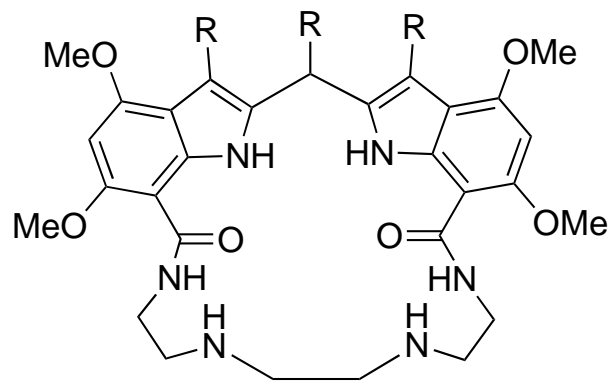

14
9

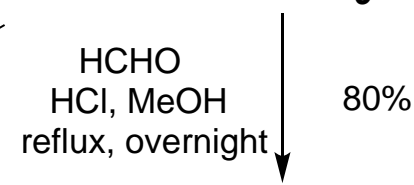

12

Scheme 4. Formation of diamide 9 and macrocyclic diindolylmethane 12.<smiles>[R]c1c[nH]c2c(C(=O)C(Cl)(Cl)Cl)c(OC)cc(OC)c12</smiles><smiles>NCCOCCOCCN</smiles>

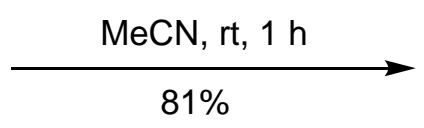

$\mathrm{R}=4-\mathrm{ClC}_{6} \mathrm{H}_{4}$

10

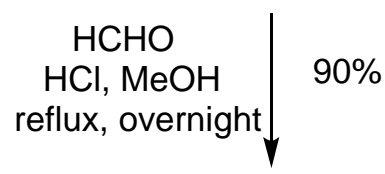<smiles>[R]c1c(Cc2[nH]c3c(C(=O)C(Cl)(Cl)C(Cl)(Cl)Cl)c(OC)cc(OC)c3c2[R])[nH]c2c(C(=O)C(Cl)(Cl)Cl)c(OC)cc(OC)c12</smiles><smiles>NCCOCCOCCN</smiles><smiles></smiles>

Scheme 5. Formation of diamide $\mathbf{1 0}$ and macrocyclic diindolylmethane $\mathbf{1 3 .}$ 


\section{Conclusions}

The synthesis of the macrocyclic diindolylmethanes 5, 6, 11, 12 and 13 indicates that diindolylmethane formation is an effective protocol for macrocyclization, as 18-, 21-, and 24-membered rings were formed. However, attempts to generate similar macrocyclic structures by ester and amide formation from diindolylmethane $\mathbf{7}$ were unsuccessful.

\section{Experimental Section}

General. ${ }^{1} \mathrm{H}$ and ${ }^{13} \mathrm{C}$ NMR spectra were recorded on a Bruker AC300F $\left({ }^{1} \mathrm{H}: 300 \mathrm{MHz},{ }^{13} \mathrm{C}: 75.5 \mathrm{MHz}\right)$ or a Bruker AM500 spectrometer. The chemical shifts $(\delta)$ and coupling constants $(J)$ are expressed in ppm and Hertz, respectively. Carbon attribution $\mathrm{C}, \mathrm{CH}, \mathrm{CH}_{2}$ and $\mathrm{CH}_{3}$ were determined by ${ }^{13} \mathrm{C}$, DEPT and $\mathrm{HMQC}$ experiments. Infrared (IR) spectra were recorded on a Mattson Genesis Series FTIR spectrometer using potassium bromide disks, except where specified. Ultraviolet and visible (UV/Vis) spectra were recorded in chloroform using a Carey 100 spectrometer. Mass spectra were recorded on a VG Quattro MS (EI) or a Finnigan MAT (MALDI). High resolution mass spectrometry (HRMS) was carried out at the Research School of Chemistry, Australian National University. Melting points were measured using a Mel-Temp melting point apparatus. Microanalyses were performed at the UNSW Microanalytical Unit and at the Campbell Microanalytical Laboratory, University of Otago, New Zealand. Column chromatography was carried out using Merck 230-400 mesh silica gel or Merck 70-230 mesh silica gel, whilst preparative TLC was performed using Merck 60GF 254 silica gel.

Ethylene bis-[3-(4-chlorophenyl)-4,6-dimethoxyindole-7-carboxylate] (2). The mixture of 7-trichloroacetylindole $1^{21}(50.0 \mathrm{mg}, 0.10 \mathrm{mmol})$, ethylene glycol $(3.58 \mathrm{mg}, 0.05 \mathrm{mmol})$ and potassium carbonate (24.0 $\mathrm{mg}, 0.17 \mathrm{mmol})$ in acetone $(10 \mathrm{~mL})$ was heated under reflux overnight, then was allowed to cool to room temperature and acidified with $\mathrm{HCl}(0.1 \mathrm{~N})$. The resulting precipitate was dissolved in dichloromethane, washed with water then dried $\left(\mathrm{MgSO}_{4}\right)$. The solvent was concentrated and the residue chromatographed using ethyl acetate as eluent to afford the title compound 2 as colorless crystals ( $30 \mathrm{mg}, 75 \%), \mathrm{mp} 252^{\circ} \mathrm{C}$ (EtOAC). IR $\left(V_{\max }, \mathrm{cm}^{-1}\right): 3400,1710,1660,1570,1370,1350,1300,1240,1200,1140,1120$. UV/Vis $\left(\lambda_{\max }, \mathrm{nm}, \varepsilon, \mathrm{cm}^{-1} \mathrm{M}^{-1}\right)$ : 246 (47,600), $312(32,300) .{ }^{1} \mathrm{H}$ NMR (300 MHz, $\left.\mathrm{CDCl}_{3}\right): \delta_{\mathrm{H}} 3.88,3.92$ (each $\left.6 \mathrm{H}, 2 \mathrm{~s}, \mathrm{OMe}\right), 4.76\left(4 \mathrm{H}, \mathrm{s}, \mathrm{CH}_{2}\right), 6.28$ $(2 \mathrm{H}, \mathrm{s}, \mathrm{H} 5), 6.95(2 \mathrm{H}, \mathrm{d}, J 2.6 \mathrm{~Hz}, \mathrm{H} 2), 7.29-7.46(8 \mathrm{H}, \mathrm{m}, \mathrm{aryl}), 10.26(2 \mathrm{H}, \mathrm{br} \mathrm{s}, \mathrm{NH}) .{ }^{13} \mathrm{C} \mathrm{NMR}\left(75 \mathrm{MHz}, \mathrm{CDCl}_{3}\right): \delta_{\mathrm{C}}$ 55.3, 57.4 (OMe), $62.3\left(\mathrm{CH}_{2}\right), 89.0$ (C5), 95.4, 110.7, 117.6 (aryl C), 121.7 (C2), 127.6, 130.8 (aryl CH), 131.8, 134.2, 139.1, 159.1, $160.8(\operatorname{aryl} C), 166.1(\mathrm{CO}) . \mathrm{MS}(+\mathrm{El}, \mathrm{m} / \mathrm{z}, \%): 691\left(\mathrm{M}^{+}+2, \mathrm{C1}^{37 / 37}, 7\right), 690\left(\mathrm{M}^{+}+1, \mathrm{Cl}^{35 / 35}, 12\right)$, 688 (20), 360 (15), 358 (50), 315 (40), 313 (100). Anal. calcd for $\mathrm{C}_{36} \mathrm{H}_{30} \mathrm{Cl}_{2} \mathrm{~N}_{2} \mathrm{O}_{8} \cdot 2 \mathrm{H}_{2} \mathrm{O}: \mathrm{C}, 59.6 ; \mathrm{H}, 4.7 ; \mathrm{N}, 3.9$. Found: C, 59.6; H, 4.4; N, 3.9\%.

Tetraethyleneglycol bis-4,6-dimethoxy-3-(4-chlorophenyl)indole-7-carboxylate (4). This compound was prepared according to the method of preparation of compound 2 using indole $1(0.20 \mathrm{~g}, 0.45 \mathrm{mmol})$, tetraethylene glycol $(43.0 \mathrm{~g}, 0.23 \mathrm{mmol})$ and potassium carbonate $(0.10 \mathrm{~g}, 0.76 \mathrm{mmol})$ in acetone $(10 \mathrm{~mL})$. The residue was chromatographed (EtOAc as eluent) to afford the title compound 4 (100 mg, 56\%) as colorless crystals, $\mathrm{mp} 220^{\circ} \mathrm{C}\left(\mathrm{CH}_{2} \mathrm{Cl}_{2}\right.$-light petroleum). IR $\left(\mathrm{V}_{\max }, \mathrm{cm}^{-1}\right)$ : 3360, 1660, 1590, 1370, 1260, 1250, 1110. UV/Vis $\left(\lambda_{\max }, \mathrm{nm}, \varepsilon, \mathrm{cm}^{-1} \mathrm{M}^{-1}\right): 207(7,400), 284(3,200) .{ }^{1} \mathrm{H}$ NMR $\left(300 \mathrm{MHz}, \mathrm{CDCl}_{3}\right): \delta_{\mathrm{H}} 3.62\left(4 \mathrm{H}, \mathrm{br} \mathrm{s}, \mathrm{CH}_{2}\right), 3.72(4 \mathrm{H}, \mathrm{br}$ $\left.\mathrm{s}, \mathrm{CH}_{2}\right), 3.78\left(4 \mathrm{H}, \mathrm{t}, J 4.6 \mathrm{~Hz}, \mathrm{CH}_{2}\right), 3.85,3.95$ (each $\left.6 \mathrm{H}, 2 \mathrm{~s}, \mathrm{OMe}\right), 4.55\left(4 \mathrm{H}, \mathrm{t}, J 4.6 \mathrm{~Hz}, \mathrm{CH}_{2}\right), 6.24(2 \mathrm{H}, \mathrm{s}, \mathrm{H} 5)$, $7.06(2 \mathrm{H}, \mathrm{d}, J 2.6 \mathrm{~Hz}, \mathrm{H} 2), 7.25-7.29(4 \mathrm{H}, \mathrm{m}, \operatorname{aryl}), 7.43-7.46(4 \mathrm{H}, \mathrm{m}, \operatorname{aryl}), 10.17(2 \mathrm{H}, \mathrm{br} \mathrm{s}, \mathrm{NH}) .{ }^{13} \mathrm{C}$ NMR $(75$ $\left.\mathrm{MHz}, \mathrm{CDCl}_{3}\right): \delta_{\mathrm{C}} 55.2,57.2(\mathrm{OMe}), 62.8,69.1,70.4,70.7\left(\mathrm{CH}_{2}\right), 88.9(\mathrm{C} 5), 121.9(\mathrm{C} 2), 127.7,130.7$ (aryl $\left.\mathrm{CH}\right)$, 
95.9, 110.5, 117.3, 131.6, 134.3, 138.8, 158.7, 160.6 (aryl C), 165.5 (CO). MS (MALDI, m/z, \%): 821 (M+, 16), 819 (25), 804 (25), 298 (100). Satisfactory microanalysis could not be obtained.

\section{4,30-Di(4-chlorophenyl)-6,8,26,28-tetramethoxy-11,14,17,20,23-pentaoxa-31,34-diazapentacyclo-}

$\left[23 \cdot 5 \cdot 2 \cdot 2^{3,9} \cdot 0^{5,13} \cdot 0^{29,32}\right]$ tetratriaconta-1(30),3,5,7,9(33),26,28,25(32)-octadecena-10,24-dione (5). The mixture of compound 4 (60 mg, $0.07 \mathrm{mmol})$, formaldehyde $(40 \%, 1 \mathrm{~mL})$ and methanol $(10 \mathrm{~mL})$ containing conc. $\mathrm{HCl}(0.5$ $\mathrm{mL}$ ) was heated under reflux overnight. After allowing the mixture to cool to room temperature, the resulting precipitate was filtered off and dried to yield the title compound 5 (50 mg, 82\%) as colorless crystals, mp 240 ${ }^{\circ} \mathrm{C}\left(\mathrm{CH}_{2} \mathrm{Cl}_{2}\right.$-light petroleum). IR ( $\left.V_{\max }, \mathrm{cm}^{-1}\right): 3380,1700,1665,1580,1370,1260,1210,1085,995$. UV/Vis $\left(\lambda_{\max }\right.$, $\left.\mathrm{nm}, \varepsilon, \mathrm{cm}^{-1} \mathrm{M}^{-1}\right): 206$ (21,200), 241 (21,000), 306 (10,700). ${ }^{1} \mathrm{H}$ NMR $\left(300 \mathrm{MHz}, \mathrm{CDCl}_{3}\right): \delta_{\mathrm{H}} 3.38\left(4 \mathrm{H}, \mathrm{br}, \mathrm{CH}_{2}\right)$, $3.40\left(4 \mathrm{H}, \mathrm{br} \mathrm{s}, \mathrm{CH}_{2}\right), 3.68\left(4 \mathrm{H}, \mathrm{br} \mathrm{s}, \mathrm{CH}_{2}\right), 3.72,3.97$ (each $\left.6 \mathrm{H}, 2 \mathrm{~s}, \mathrm{OMe}\right), 4.24\left(2 \mathrm{H}, \mathrm{s}, \mathrm{CH}_{2}\right), 4.46(4 \mathrm{H}, \mathrm{t}, J 4.6 \mathrm{~Hz}$, $\left.\mathrm{CH}_{2}\right), 6.20(2 \mathrm{H}, \mathrm{s}, \mathrm{H} 5), 7.09(8 \mathrm{H}, \mathrm{s}, \mathrm{aryl}), 9.82(2 \mathrm{H}, \mathrm{s}, \mathrm{NH}) .{ }^{13} \mathrm{C} \mathrm{NMR}\left(75 \mathrm{MHz}, \mathrm{CDCl}_{3}\right): \delta_{\mathrm{C}} 24.4,62.0,68.4,69.6$, $69.8\left(\mathrm{CH}_{2}\right), 55.2,57.2$ (OMe), 88.7 (C5), 95.7, 112.0, 113.8 (aryl C), 126.8, 131.8 (aryl CH), 130.0, 131.4, 133.6, 137.0, 158.2, 160.2 (aryl C), 164.6 (CO). MS (+El, $m / z, \%): 855\left(\mathrm{M}^{+}+\mathrm{Na}, 80\right)$. Anal. calcd for $\mathrm{C}_{43} \mathrm{H}_{42} \mathrm{Cl}_{2} \mathrm{~N}_{2} \mathrm{O}_{11} \cdot 0.5 \mathrm{H}_{2} \mathrm{O}: \mathrm{C}, 61.3 ; \mathrm{H}, 5.1 ; \mathrm{N}, 3.3$. Found: $\mathrm{C}, 61.3 ; \mathrm{H}, 5.1 ; \mathrm{N}, 3.3 \%$.

\section{2,4,30-Tri(4-chlorophenyl)-6,8,26,28-tetramethoxy-11,14,17,20,23-pentaoxa-31,34-diazapentacyclo-}

$\left[23 \cdot 5 \cdot 2 \cdot 2^{3,9} \cdot 0^{5,13} \cdot 0^{29,32}\right]$ tetratriaconta-1(30),3,5,7,9(33),26,28,25(32)-octadecena-10,24-dione (6). This compound was prepared according to the method of preparation of compound 5 using compound 4 (100 mg, $0.12 \mathrm{mmol}$ ) and 4-chlorobenzaldehyde $(8.5 \mathrm{mg}, 0.06 \mathrm{mmol})$ in methanol $(10 \mathrm{~mL})$ containing conc. $\mathrm{HCl}(0.5 \mathrm{~mL})$. The reaction mixture was heated under reflux for $3 \mathrm{~h}$. Water was added to the cooled reaction mixture and the resulting precipitate was filtered off and triturated with dichloromethane and light petroleum to yield the title compound 6 (30 mg, 53\%) as a colorless solid, $\mathrm{mp} 180{ }^{\circ} \mathrm{C}$. IR ( $\left.\mathrm{V}_{\mathrm{max}} \mathrm{cm}^{-1}\right)$ : 3400, 1720, 1655, 1590, 1370, 1270, 1210, 1160. UV/Vis $\left(\lambda_{\max }, \mathrm{nm}, \varepsilon, \mathrm{cm}^{-1} \mathrm{M}^{-1}\right): 206(29,800), 223(28,500), 303(11,500) .{ }^{1} \mathrm{H} \mathrm{NMR}(300 \mathrm{MHz}$, $\left.\mathrm{CDCl}_{3}\right): \delta_{\mathrm{H}} 3.55\left(4 \mathrm{H}, \mathrm{br} \mathrm{s}, \mathrm{CH}_{2}\right), 3.59\left(4 \mathrm{H}, \mathrm{br} \mathrm{s}, \mathrm{CH}_{2}\right), 3.85\left(4 \mathrm{H}, \mathrm{br} \mathrm{s}, \mathrm{CH}_{2}\right), 3.71,3.95$ (each $\left.6 \mathrm{H}, 2 \mathrm{~s}, \mathrm{OMe}\right), 4.46(4 \mathrm{H}$, t, J $\left.5.1 \mathrm{~Hz}, \mathrm{CH}_{2}\right), 5.63(1 \mathrm{H}, \mathrm{s}, \mathrm{CH}), 6.23(2 \mathrm{H}, \mathrm{s}, \mathrm{H} 5), 6.95-7.14(12 \mathrm{H}, \mathrm{m}, \operatorname{aryl}), 9.93(2 \mathrm{H}, \mathrm{s}, \mathrm{NH}) .{ }^{13} \mathrm{C} \mathrm{NMR}(75 \mathrm{MHz}$, $\mathrm{CDCl}_{3}$ ): $\delta_{\mathrm{C}} 39.2(\mathrm{CH}), 55.2,57.4$ (OMe), 63.5, 69.0, 70.4, $70.6\left(\mathrm{CH}_{2}\right), 89.4(\mathrm{C} 5), 96.0,111.8,114.9$ (aryl C), 127.4, 129.4, 129.5, 131.6 (aryl CH), 130.7, 132.1, 133.1, 138.3, 138.4, 139.3, 158.6, 160.5 (aryl C), 165.7 (CO). MS (MALDI, $m / z, \%): 943\left(\mathrm{M}^{+}-1,50\right)$. Anal. calcd for $\mathrm{C}_{49} \mathrm{H}_{45} \mathrm{Cl}_{3} \mathrm{~N}_{2} \mathrm{O}_{11}$ : C, 62.3; $\mathrm{H}, 4.8 ; \mathrm{N}, 3.0$. Found: $\mathrm{C}, 62.8 ; \mathrm{H}, 4.7 ; \mathrm{N}$, $3.2 \%$.

3-Azapentylene bis-3-(4-chlorophenyl)-4,6-dimethoxyindole-7-carboxamide (8). A solution of indole 1 (0.40 $\mathrm{g}, 0.90 \mathrm{mmol})$ in anhydrous acetonitrile $(20 \mathrm{~mL})$ was treated with triethylenediamine $(0.50 \mathrm{~g}, 0.50 \mathrm{mmol})$ and the solution was stirred at room temperature for $1 \mathrm{~h}$. The resulting precipitate was filtered off, washed with acetonitrile and dried to afford the title compound $8(0.40 \mathrm{~g}, 95 \%)$ as yellow crystals, $\mathrm{mp} 266{ }^{\circ} \mathrm{C}\left(\mathrm{CH}_{2} \mathrm{Cl}_{2}\right)$. IR $\left(V_{\max }, \mathrm{cm}^{-1}\right): 3400,3360,3340,1620,1590,1370,1340,1620,1200,1210,1150,1100$. UV/Vis $\left(\lambda_{\max }, \mathrm{nm}, \varepsilon, \mathrm{cm}^{-}\right.$ $\left.{ }^{1} \mathrm{M}^{-1}\right): 239$ (30,100), $211(25,200), 308(16,800) .{ }^{1} \mathrm{H} \mathrm{NMR}\left(300 \mathrm{MHz}, \mathrm{CDCl}_{3}\right): \delta_{\mathrm{H}} 2.98\left(4 \mathrm{H}, \mathrm{t}, J 6.2 \mathrm{~Hz}, \mathrm{CH}_{2}\right), 3.61$ $\left(2 \mathrm{H}, \mathrm{q}, J 5.6 \mathrm{~Hz}, \mathrm{CH}_{2}\right), 3.76,3.86$ (each 6H, 2s, OMe), $6.03(2 \mathrm{H}, \mathrm{s}, \mathrm{H} 5), 7.09(2 \mathrm{H}, \mathrm{d}, J 2.1 \mathrm{~Hz}, \mathrm{H} 2), 7.31(4 \mathrm{H}, \mathrm{d}, J 6.6$ $\mathrm{Hz}$, aryl), $7.47(4 \mathrm{H}, \mathrm{d}, J 8.8 \mathrm{~Hz}, \operatorname{aryl}), 8.38(2 \mathrm{H}, \mathrm{t}, J 5.2 \mathrm{~Hz}, \mathrm{NH}), 11.18(2 \mathrm{H}, \mathrm{br}, \mathrm{NH})$. One NH signal did not appear. ${ }^{13} \mathrm{C}$ NMR $\left(75 \mathrm{MHz}, \mathrm{CDCl}_{3}\right): \delta_{\mathrm{C}} 36.9\left(\mathrm{CH}_{2}\right), 55.2,56.8(\mathrm{OMe}), 65.0\left(\mathrm{CH}_{2}\right), 87.4(\mathrm{C} 5), 105.8,121.4(\operatorname{aryl~C})$, 121.9 (C2), 127.7 (aryl CH), 128.2 (aryl C), 130.7 (aryl CH), 131.5, 134.5, 139.6, 156.1, 157.0 (aryl C), 167.8 (CO). $\mathrm{MS}$ (+El, m/z, \%): 732 (M, 70), 731 (75), 730 (100). Anal. calcd for $\mathrm{C}_{38} \mathrm{H}_{37} \mathrm{Cl}_{2} \mathrm{~N}_{5} \mathrm{O}_{6} \cdot 1.1 \mathrm{CH}_{2} \mathrm{Cl}_{2}: \mathrm{C}, 57.0 ; \mathrm{H}, 4.8 ; \mathrm{N}$, 8.5. Found: $C, 56.9 ; H, 5.0 ; N, 8.6 \%$.

3,6-Diazaoctylene bis-3-(4-chloropheny1)-4,6-dimethoxyindole-7-carboxamide (9). This compound was prepared according to the method of preparation of compound 8 using indole $1(0.10 \mathrm{~g}, 0.23 \mathrm{mmol})$ and tetraethylenetriamine $(17.0 \mathrm{mg}, 0.12 \mathrm{mmol})$. The resulting precipitate was filtered off and dried to afford the title compound 9 (50.0 mg, 60\%) as yellow crystals, $\mathrm{mp} 262{ }^{\circ} \mathrm{C}\left(\mathrm{CH}_{2} \mathrm{Cl}_{2}-\mathrm{MeOH}\right) . \mathrm{IR}\left(\mathrm{V}_{\max }, \mathrm{cm}^{-1}\right): 3400,3300$, 
3320, 1620, 1595, 1580, 1330, 1255, 1215. UV/Vis $\left(\lambda_{\max }, \mathrm{nm}, \varepsilon, \mathrm{cm}^{-1} \mathrm{M}^{-1}\right): 240(40,700), 307(25,400) .{ }^{1} \mathrm{H} N M R$ (300 MHz, CDCl 3$): \delta_{\mathrm{H}} 1.84\left(2 \mathrm{H}\right.$, br s, NH), $2.87\left(4 \mathrm{H}, \mathrm{br} \mathrm{s}, \mathrm{CH}_{2}\right), 2.94\left(4 \mathrm{H}, \mathrm{t}, J 6.2 \mathrm{~Hz}, \mathrm{CH}_{2}\right), 3.59\left(4 \mathrm{H}, \mathrm{br} \mathrm{s}, \mathrm{CH}_{2}\right)$, 3.64, 3.92 (each 6H, 2s, OMe), $5.96(2 \mathrm{H}, \mathrm{s}, \mathrm{H} 5), 7.07(2 \mathrm{H}, \mathrm{d}, \mathrm{J} 2.1 \mathrm{~Hz}, \mathrm{H} 2), 7.25-7.28(4 \mathrm{H}, \mathrm{m}, \operatorname{aryl}), 7.43-7.46(4 \mathrm{H}$, $\mathrm{m}$, aryl), $8.45\left(2 \mathrm{H}\right.$, br s, NH), $11.18(2 \mathrm{H}, \mathrm{br} \mathrm{s}, \mathrm{NH}) .{ }^{13} \mathrm{C} \mathrm{NMR}\left(75 \mathrm{MHz}, \mathrm{CDCl}_{3}\right): \delta_{\mathrm{C}} 39.2,48.2,49.1\left(\mathrm{CH}_{2}\right), 54.9,56.8$ (OMe), 87.3 (C5), 97.6, 108.6, 116.8 (aryl C), 121.9 (C2), 127.7, 130.7 (aryl CH), 131.5, 134.4, 139.5, 156.7, 157.7 (aryl C), 167.9 (CO). MS (MALDI, $m / z, \%): 773$ (M, 100). Anal. calcd for $\mathrm{C}_{40} \mathrm{H}_{42} \mathrm{Cl}_{2} \mathrm{~N}_{6} \mathrm{O}_{6} .0 .5 \mathrm{H}_{2} \mathrm{O}: \mathrm{C}, 61.4 ; \mathrm{H}$, 5.5; N, 10.7. Found: C, 61.2; $H, 5.2 ; \mathrm{N}, 10.6 \%$.

3,6-Dioxaoctylene bis-3-(4-chlorophenyl)-4,6-dimethoxyindole-7-carboxamide (10). A solution of indole 1 $(0.53 \mathrm{~g}, 1.22 \mathrm{mmol})$ in anhydrous acetonitrile $(20 \mathrm{~mL})$ was treated with 2,2'-(ethylenedioxy)bis(ethylamine) $(0.50 \mathrm{~g}, 0.45 \mathrm{mmol})$ and the solution was stirred at room temperature for $1 \mathrm{~h}$. The resulting precipitate was filtered off, washed with acetonitrile and dried to afford the title compound $\mathbf{1 0}(0.40 \mathrm{~g}, 81 \%)$ as a yellow solid, $\mathrm{mp} 224^{\circ} \mathrm{C} . \mathrm{IR}\left(v_{\max }, \mathrm{cm}^{-1}\right)$ : 3400, 3340, 1620, 1590, 1525, 1350, 1335, 1250, 1210, 1100, 975. UV/Vis $\left(\lambda_{\max }, \mathrm{nm}\right.$, $\left.\varepsilon, \mathrm{cm}^{-1} \mathrm{M}^{-1}\right): 244(35,800), 305$ (31,000). ${ }^{1} \mathrm{H}$ NMR $\left(300 \mathrm{MHz}, \mathrm{CDCl}_{3}\right): \delta_{\mathrm{H}} 3.52\left(4 \mathrm{H}, \mathrm{br} \mathrm{s}, \mathrm{CH}_{2}\right), 3.69-3.74(14 \mathrm{H}, \mathrm{m}$, $\mathrm{CH}_{2}$ \& OMe ), $3.87(6 \mathrm{H}, \mathrm{s}, \mathrm{OMe}), 5.82(2 \mathrm{H}, \mathrm{s}, \mathrm{H} 5), 7.08(2 \mathrm{H}, \mathrm{d}, J 2.1 \mathrm{~Hz}, \mathrm{H} 2), 7.27(4 \mathrm{H}, \mathrm{d}, J 8.7 \mathrm{~Hz}, \operatorname{aryl}), 7.45(4 \mathrm{H}$, d, J $8.7 \mathrm{~Hz}$, aryl), $8.52\left(2 \mathrm{H}\right.$, br s, NH), $11.70\left(2 \mathrm{H}\right.$, br s, NH). ${ }^{13} \mathrm{C} \mathrm{NMR}\left(75 \mathrm{MHz}, \mathrm{CDCl}_{3}\right): \delta_{\mathrm{C}} 39.2\left(\mathrm{CH}_{2}\right), 54.7,56.7$ (OMe), 70.0, 70.6 ( $\left.\mathrm{CH}_{2}\right), 87.2$ (C5), 97.2, 110.7, 116.9 (aryl C), 121.7 (C2), 127.7, 130.7 (aryl CH), 131.6, 134.4, 139.4, 156.9, 157.1 (aryl C), 167.7 (CO). MS (+El, m/z, \%): $776\left(\mathrm{M}^{+}+1,75\right), 556$ (60), 442 (100). Anal. calcd for $\mathrm{C}_{40} \mathrm{H}_{40} \mathrm{Cl}_{2} \mathrm{~N}_{4} \mathrm{O}_{8} \cdot 0.5 \mathrm{H}_{2} \mathrm{O}: \mathrm{C}, 61.2 ; \mathrm{H}, 5.3 ; \mathrm{N}$ 7.1. Found: $\mathrm{C}, 61.0 ; \mathrm{H}, 5.2 ; \mathrm{N}, 7.0 \%$.

\section{4,24-Di(4-chlorophenyl)-6,8,20,22-tetramethoxy-11,14,17,26,28-pentaaza-pentacyclo-}

$\left[17.5 \cdot 2 \cdot 2^{3,9} \cdot 0^{5,27} \cdot 0^{23,26}\right]$ octacosa-1(24),3,5,7,9(27),19(26),20,22-octadecene-10,18-dione (11). A mixture of compound $8(62.0 \mathrm{mg}, 0.08 \mathrm{mmol})$, formaldehyde $(40 \%, 4 \mathrm{~mL})$ and methanol $(10 \mathrm{~mL})$ containing conc. $\mathrm{HCl}(0.5$ $\mathrm{mL}$ ) was heated under reflux overnight. After allowing the mixture to cool to room temprerature, the resulting precipitate was filtered off and dried to yield the title compound 11 (46.0 mg, 74\%) as colorless crystals, mp $252{ }^{\circ} \mathrm{C}\left(\mathrm{CH}_{2} \mathrm{Cl}_{2}-\mathrm{MeOH}\right)$. IR $\left(v_{\max }, \mathrm{cm}^{-1}\right)$ : 3495, 1620, 1595, 1370, 1270, 1200, 1100, 991. UV/Vis $\left(\lambda_{\max }, \mathrm{nm}, \varepsilon, \mathrm{cm}^{-}\right.$ $\left.{ }^{1} \mathrm{M}^{-1}\right)$ : $317(20,800) .{ }^{1} \mathrm{H}$ NMR $(300 \mathrm{MHz}$, DMSO-d $): \delta_{\mathrm{H}} 3.19\left(4 \mathrm{H}, \mathrm{br} \mathrm{s}, \mathrm{CH}_{2}\right), 3.71\left(4 \mathrm{H}, \mathrm{br} \mathrm{s}, \mathrm{CH}_{2}\right), 3.73,3.98($ each $6 \mathrm{H}, 2 \mathrm{~s}, \mathrm{OMe}), 4.11\left(2 \mathrm{H}, \mathrm{s}, \mathrm{CH}_{2}\right), 6.39(2 \mathrm{H}, \mathrm{s}, \mathrm{H} 5), 6.98(4 \mathrm{H}, \mathrm{d}, J 8.2 \mathrm{~Hz}, \operatorname{aryl}), 7.19(4 \mathrm{H}, \mathrm{d}, J 8.2 \mathrm{~Hz}, \operatorname{aryl}), 8.62(1 \mathrm{H}$, s, NH), $8.95\left(2 \mathrm{H}\right.$, br s, NH), $11.93(2 \mathrm{H}, \mathrm{br} \mathrm{s}, \mathrm{NH}) .{ }^{13} \mathrm{C} \mathrm{NMR}\left(75 \mathrm{MHz}^{\mathrm{CDCl}} \mathrm{CD}_{3}\right): \delta_{\mathrm{C}} 40.4\left(\mathrm{CH}_{2}\right), 53.4,54.9$ (OMe), 56.4, $70.4\left(\mathrm{CH}_{2}\right), 86.1$ (C5), 94.7, 108.8, 109.2 (aryl C), 125.1 (aryl CH), 128.5, 130.16 (aryl C), 130.22 (aryl CH), 131.9, 135.4, 154.4, 154.7 (aryl C), 166.2 (CO). MS (+El, m/z, \%): $744\left(\mathrm{M}^{+}, \mathrm{Cl}^{35135}, 75\right), 743$ (77), 742 (100). Anal. calcd for $\mathrm{C}_{39} \mathrm{H}_{37} \mathrm{Cl}_{2} \mathrm{~N}_{5} \mathrm{O}_{6} \cdot \mathrm{CH}_{2} \mathrm{Cl}_{2}$ : C, 55.9; $\mathrm{H}, 4.6 ; \mathrm{N}, 8.1$. Found: $\mathrm{C}, 55.9 ; \mathrm{H}, 4.9 ; \mathrm{N}, 8.1 \%$.

\section{4,27-Di(4-chlorophenyl)-6,8,23,25-tetramethoxy-11,14,17,20,28,31-hexaaza-pentacyclo-}

$\left[20.5 \cdot 2 \cdot 2^{3,9} \cdot 0^{5,30} \cdot 0^{26,29}\right]$ hentriconta $1(27), 3,5,7,9(30), 23,25,29(22)$ octadecene-10,21-dione (12). This compound was prepared according to the method of preparation of compound 11 using compound 9 (40.0 mg, 0.05 $\mathrm{mmol})$ and formaldehyde $(40 \%, 1.0 \mathrm{~mL})$ in methanol $(10 \mathrm{~mL})$ containing conc. $\mathrm{HCl}(1 \mathrm{~mL})$. After allowing the mixture to cool to room temperature, sat. $\mathrm{NaHCO}_{3}$ was added to the mixture until basic. The mixture was extracted with EtOAc and the organic layer washed with water, and dried $\left(\mathrm{MgSO}_{4}\right)$. The solvent was evaporated off to yield the title compound $12(24 \mathrm{mg}, 80 \%)$ as pale yellow crystals, $\mathrm{mp} 246{ }^{\circ} \mathrm{C}\left(\mathrm{CH}_{2} \mathrm{Cl}_{2}\right.$-light petroleum). IR ( $\left.V_{\max }, \mathrm{cm}^{-1}\right): 3380,1620,1590,1260,1210 . \mathrm{UV} / \mathrm{Vis}\left(\lambda_{\max } \mathrm{nm}, \varepsilon, \mathrm{cm}^{-1} \mathrm{M}^{-1}\right): 238(39,700), 304$ (20,600). ${ }^{1} \mathrm{H}$ NMR $\left(300 \mathrm{MHz}, \mathrm{CDCl}_{3}\right): \delta_{\mathrm{H}} 2.81\left(4 \mathrm{H}, \mathrm{t}, J 6.2 \mathrm{~Hz}, \mathrm{CH}_{2}\right), 2.87\left(4 \mathrm{H}, \mathrm{br} \mathrm{s}, \mathrm{CH}_{2}\right), 3.64\left(4 \mathrm{H}, \mathrm{t}, J 6.2 \mathrm{~Hz}, \mathrm{CH}_{2}\right)$, 3.72, 3.97 (each $6 \mathrm{H}, 2 \mathrm{~s}, \mathrm{OMe}), 4.10\left(2 \mathrm{H}, \mathrm{s}, \mathrm{CH}_{2}\right), 6.17(2 \mathrm{H}, \mathrm{s}, \mathrm{H} 5), 7.08-7.20(8 \mathrm{H}, \mathrm{m}, \operatorname{aryl}), 7.26(2 \mathrm{H}, \mathrm{br} \mathrm{s}, \mathrm{NH})$, $7.96(2 \mathrm{H}, \mathrm{t}, \mathrm{J} 6.2 \mathrm{~Hz}, \mathrm{NH}), 11.51(2 \mathrm{H}, \mathrm{s}, \mathrm{NH}) .{ }^{13} \mathrm{C} \mathrm{NMR}\left(75 \mathrm{MHz}, \mathrm{CDCl}_{3}\right): \delta_{\mathrm{C}} 23.3,38.8,53.6\left(\mathrm{CH}_{2}\right), 55.2(\mathrm{OMe})$, $55.9\left(\mathrm{CH}_{2}\right), 56.9$ (OMe), 87.7 (C5), 97.9, 112.3, 112.5 (aryl C), 127.4 (aryl CH), 131.1, 131.6, 132.1 (aryl C), 133.8 (aryl CH), 138.6, 156.1, 156.6 (aryl C), $167.6 \mathrm{CO}) . \mathrm{MS}(+\mathrm{El}, \mathrm{m} / z, \%): 785\left(\mathrm{M}^{+}, \mathrm{C} 1^{35 / 35}, 100\right)$. Anal. calcd for $\mathrm{C}_{41} \mathrm{H}_{42} \mathrm{Cl}_{2} \mathrm{~N}_{6} \mathrm{O}_{6} \cdot 1.7 \mathrm{CH}_{2} \mathrm{Cl}_{2}: \mathrm{C}, 55.1 ; \mathrm{H}, 4.9 ; \mathrm{N}, 9.0$. Found: $\mathrm{C}, 55.1 ; \mathrm{H}, 5.1 ; \mathrm{N}, 8.5 \%$. 


\section{4,27-Di(4-chlorophenyl)-6,8,23,25-tetramethoxy-14,17-dioxa-11,20,29,31-} tetraazapentacyclo[20.5.2.2 $\left.2^{3,9} \cdot 0^{5,30} \cdot 0^{26,29}\right]$ hentriaconta-1(27),3,5,7,9(30),22(29),23,25-octadecene-10,24-

dione (13). This compound was prepared according to the method of preparation of compound 11 using compound $10(0.20 \mathrm{~g}, 0.26 \mathrm{mmol})$ and formaldehyde $(40 \%, 2 \mathrm{~mL})$ in methanol $(10 \mathrm{~mL})$ containing conc. $\mathrm{HCl}(1$ $\mathrm{mL})$. The resulting precipitate was filtered off and dried to yield the title compound $13(0.30 \mathrm{~g}, 90 \%)$ as colorless crystals, $\mathrm{mp} 275^{\circ} \mathrm{C}\left(\mathrm{CH}_{2} \mathrm{Cl}_{2}-\mathrm{MeOH}\right)$. IR $\left(v_{\max } \mathrm{cm}^{-1}\right): 3420,3320,1630,1590,1360,1300,1260,1200$, 995. UV/Vis $\left(\lambda_{\max }, \mathrm{nm}, \varepsilon, \mathrm{cm}^{-1} \mathrm{M}^{-1}\right): 206(10,207), 302(4,462) .{ }^{1} \mathrm{H} \mathrm{NMR}\left(300 \mathrm{MHz}, \mathrm{CDCl}_{3}\right): \delta_{\mathrm{H}} 3.58\left(4 \mathrm{H}, \mathrm{br} \mathrm{s}, \mathrm{CH}_{2}\right)$, $3.62\left(4 \mathrm{H}\right.$, br s, $\left.\mathrm{CH}_{2}\right), 3.76\left(4 \mathrm{H}, \mathrm{br} \mathrm{s}, \mathrm{CH}_{2}\right), 3.69,3.99$ (each $\left.6 \mathrm{H}, \mathrm{s}, \mathrm{OMe}\right), 4.11\left(2 \mathrm{H}, \mathrm{s}, \mathrm{CH}_{2}\right), 6.20(2 \mathrm{H}, \mathrm{s}, \mathrm{H} 5), 7.13-$ $7.27(8 \mathrm{H}, \mathrm{m}, \mathrm{aryl}), 8.05(2 \mathrm{H}, \mathrm{t}, \mathrm{J} 5.6 \mathrm{~Hz}, \mathrm{NH}), 11.33(2 \mathrm{H}, \mathrm{br} \mathrm{s}, \mathrm{NH}) .{ }^{13} \mathrm{C} \mathrm{NMR}(75 \mathrm{MHz}, \mathrm{CDCl} 3): \delta_{\mathrm{C}} 23.5,39.7\left(\mathrm{CH}_{2}\right)$, 55.2, 56.9 (OMe), 69.7, $70.4\left(\mathrm{CH}_{2}\right), 87.6$ (C5), 97.8, 112.4, 112.6 (aryl C), 127.4 (aryl CH), 131.0, 131.7 (aryl C), 132.1 (aryl CH), 133.8, 138.5, 156.1, 156.7 (aryl C), 167.8 (C0). MS (MALDI, m/z, \%): 787 (M+, 80 ), 785 (100). 771 (75). Anal. calcd for $\mathrm{C}_{41} \mathrm{H}_{40} \mathrm{Cl}_{2} \mathrm{~N}_{4} \mathrm{O}_{8} \cdot 2.5 \mathrm{H}_{2} \mathrm{O}: \mathrm{C}, 59.1 ; \mathrm{H}, 5.4 ; \mathrm{N}, 6.7$. Found: $\mathrm{C}, 59.2 ; \mathrm{H}, 5.2 ; \mathrm{N}, 7.2 \%$.

\section{Acknowledgements}

Financial support from the Australian Research Council is gratefully acknowledged. B.P. also acknowledges receipt of an International Postgraduate Research Scholarship from the Australian Government.

\section{References}

1. Dietrich, B.; Viout, P.; Lehn, J.-M. Macrocyclic Chemistry: Aspects of Organic and Inorganic Supramolecular Chemistry, 1993, (VCH Verlagsgesellschaft, Weinheim, Germany).

2. Amabilino, D. B.; Stoddart, J. F. Chem. Rev. 1995. 95, 2725-2828. https://doi.org/10.1021/cr00040a005

3. Joule, J. A. Sci. Synth. 2001, 10, 361-652. https://doi.org/10.1023/A:1017537510851

4. Yu, X.; Sun, D. Molecules 2013, 18, 6230-6268. https://doi.org/10.3390/molecules18066230

5. Driggers, E. M.; Hale, S. P.; Lee, J.; Terrett, N. K. Nat. Rev. Drug Discovery 2008, 7, 608-624. https://doi.org/10.1038/nrd2590

6. Mallinson, J.; Collins, I. Future Med. Chem. 2012, 4, 1409-1438. https://doi.org/10.4155/fmc.12.93

7. Goekjian, P. G.; Wu, G. Z.; Chen, S.; Zhou, L.; Jirousek, M. R.; Gillig, J. R.; Ballas, L. M.; Dixon, J. T. J. Org. Chem. 1999, 64, 4238-4246. https://doi.org/10.1021/jo9808876

8. Zhang, H. C.; White, K. B.; Ye, H.; McComsey, D. F.; Derian, C. K.; Addo, M. F.; Andrade-Gordon, P.; Eckardt, A. J.; Conway, B. R.; Westover, L. Bioorg. Med. Chem. Lett. 2003, 13, 3049-3053. https://doi.org/10.1016/S0960-894X(03)00644-9

9. Black, D. StC.; Rothnie, N. E.; Wong, L. C. H. Aust. J. Chem. 1983, 36, 2407-2412. https://doi.org/10.1071/CH9832407

10. Bowyer, P. K.; Black, D. StC.; Craig, D. C. Tetrahedron 2005, 61, 10781-10792. 
11. Sengul, I. F.; Wood, K.; Kumar, N.; Black, D. StC. Tetrahedron 2012, 68, 9050-9055.

https://doi.org/10.1016/j.tet.2012.08.063

12. Kandemir, H.; Sengul, I. F.; Kumar, N.; Black, D. StC. Aust. J. Chem. 2017, 70, 1196-1201.

https://doi.org/10.1071/CH17264

13. Somphol, K.; Kumar, N.; Black, D. StC. Heterocycles 2021, 102, 686-704.

https://doi.org/10.3987/COM-21-14411

14. Black, D. StC.; Craig, D. C.; Kumar, N. J. Chem. Soc., Chem. Commun. 1989, 425-426.

https://doi.org/10.1039/c39890000425

15. Black, D. StC.; Craig, D. C.; Kumar, N. Aust. J. Chem. 1996, 49, 311-318.

https://doi.org/10.1071/CH9960311

16. 1Black, D. StC.; Bowyer, M. C.; Kumar, N.; Mitchell, P. S. R. J. Chem. Soc., Chem. Commun. 1993, 819-821. https://doi.org/10.1039/c39930000819

17. Chen, R.; Somphol, K.; Bhadbhade, M.; Kumar, N.; Black, D. StC. Synlett 2013, 24, 1497-1500. https://doi.org/10.1055/s-0033-1338868

18. Dobrowolski, J. C.; Somphol, K.; Santoso, M.; Duong, H.; Gardner, C. R.; Kumar, N.; Black, D. StC. Aust. J. Chem. 2017, 70, 1188-1195.

https://doi.org/10.1071/CH17257

19. Chen, R.; Bhadbhade, M.; Kumar, N.; Black, D. StC. Tetrahedron Lett. 2012, 53, 3337-3341.

https://doi.org/10.1016/j.tetlet.2012.04.082

20. Purwono, B.; Kumar, N.; Black, D. StC. Arkivoc 2022, https://doi.org/10.24820/ark.5550190.p011.668

21. Purwono, B.; Kumar, N.; Black, D. StC. Arkivoc 2022, iv, 6-23.

https://doi.org/10.24820/ark.5550190.p011.637

22. Black, D. StC.; Bowyer, M. C.; Catalano, M. M.; Ivory, A. J.; Keller, P. A.; Kumar, N.; Nugent, S. J. Tetrahedron 1994, 50, 10497-10508.

https://doi.org/10.1016/S0040-4020(01)89590-9 